\title{
Printed Telugu Numeral Recognition based on Structural, Skeleton and Water Reservoir Features
}

\author{
U Ravi Babu ${ }^{1}$ Y V V Satyanarayana ${ }^{2}$ S. Marthu Perumal ${ }^{3}$ \\ 1 Research Scholar, ANU, Department of CSE \\ uppu.ravibabu@gmail.com \\ 2 M.Tech (CSE) Student, GIET, Rajahmundry \\ yvvsna@gmail.com \\ Assoc Professor, Department of CSE, GIET, Rajahmundry
}

\section{ABSTRACT}

Selection of feature extraction method is most important factor in achieving high recognition performance in automatic numeral recognition systems In this paper, a new proposed system for Telugu printed numeral characters recognition using number of contours, skeleton feature, water reservoir features, and ratio of length of Top line to bottom line of the image. Printed Telugu numerals are scan converted to binary images and extract the features irrespective of size of the image. The proposed method is applied with success to a database of 3150 printed multi-font printed Telugu numerals The recognition accuracy is achieved $100 \%$. The experimental results obtained are encouraging and comparable with other methods found in literature survey. The novelty of the proposed method is free from size normalization and without classification method.

\section{Indexing terms/Keywords}

Telugu numeral recognition, water reservoir, number of contors, skeleton feature

\section{Council for Innovative Research}

Peer Review Research Publishing System

\section{Journal: INTERNATIONAL JOURNAL OF COMPUTERS \& TECHNOLOGY}

Vol 10, No 7

editor@cirworld.com

www.cirworld.com, member.cirworld.com 


\section{INTRODUCTION}

Optical character recognition system is an image processing system which gets a textural image as input and after processing, it produces corresponding editable text as output. With respect to how image is acquired, OCR systems are divided into the two different categories. In offline systems, first, a textural document is scanned completely and then its image is processed while in online systems, processing is done during the text is written by optical pen on a digitized tablet. The recognition of machine numerals has been the subject of much attention in pattern recognition. The numeral character recognition has a large number of applications such as license plate recognition, automatic check ordering in banks, automatic postal code recognition of mails, processing of numeral forms interpretation of ID numbers etc. Promising feature extraction methods have been identified in the literature for recognition of characters and numerals of many different scripts. For recognition of image object one need to extract the potential features. Thus, Feature extraction plays a vital role in Pattern Recognition and Image Processing systems in general and character recognition systems in particular, Ivind and Jain [9] present a survey of various feature extraction methods used in character recognition system.

The problem of numeral recognition has been studied for decades and many methods have been proposed, e.g. template matching, dynamic programming, hidden Markov modeling, neural network, expert system and combinations of these techniques [2, 4, and 15]. Recognition of character/numeral in foreign languages like English, Chinese, Japanese, and Arabic reported many authors. In the Indian context, major research work reported in Devanagari, Tamil, Bengali, Oriya and Kannada numeral recognition system [1, 3, 7, 13, 17, 18, and 19]. Compared to the work attributed in the area of Devanagari and Bangla numerals,only few works related Kannda numeral recognition can be identified [8, 10, 11, and 23].

Promising feature extraction methods have been identified in the literature for recognition of characters and numerals of many different scripts. These include template matching, projection histograms, geometric moments, Zernike moments, contour profile, Fourier descriptors, and unitary transforms. A brief review of these feature extraction methods is found in [9]. Various methods have been proposed, and high recognition rates are reported, for the recognition of English handwritten digits $[5,6]$.

Selection of a feature extraction method is most important factor in achieving high recognition performance in character/numeral recognition systems. Different feature extraction methods have been proposed for numerals belonging to different Indian scripts. We briefly review them below. U. Pal and B. B. Chaudhuri [3] have reported a complete printed Bangla OCR system for recognition of Bangla numerals and characters. In [19] recognition of handwritten Devanagari numerals is described. Three different types of features, namely, density features, moment features and descriptive component features are used. Hanmandlu et.al [13] have proposed a fuzzy based approach to recognition of multifont numerals. The preprocessed numeral image is partitioned in to fixed number of sub images called boxes and normalized vector distance of foreground pixels are computed and used as features. Multi-font numeral recognition without thinning based on directional density of pixels is reported in [7]. The outer densities of pixels for each of the direction are computed in four directions viz. bottom, top, left and right. The ratios of these densities are taken with the total area of the cropped numeral image and are stored in a feature vector. In [8] a 10-segmentstring concept, water reservoir principle, horizontal and vertical strokes, and end points are used as features for Kannada handwritten numerals. G. G. Rajput et.al [11] have proposed to work towards recognition of Kannada numerals using Fourier descriptors obtained from the crack code of the numeral images for feature extraction and SVM classifier is used for classification. From the literature survey it is clear that most of the work done for numeral recognition includes feature extraction methods and classification methods are required to recognize the numerals. This motivated us to work towards recognition of Telugu numerals with out using any classification algorithms only based on features to recognize the numerals

The rest of the paper is described as follows. Section 2 describes data collection and preprocessing. Feature extraction is explained in section 3. Recognition method is described in section 4. Experimental results are discussed in section 5 and conclusion is given in section 6 .

\section{DATA SET AND PREPROCESSING}

\subsection{Data Collection}

Telugu, the official language of the southern Indian state of Andhra Pradesh, Telugu is a Central Dravidian language primarily spoken in the state of Andhra Pradesh, India. It is also spoken in the neighboring states of Chhattisgarh, Karnataka, Maharashtra, Orissa and Tamil Nadu, and is spoken in the bordering city of Yanam, in the neighboring territory of Pondicherry. According to the 2011 Census of India, Telugu is the language with the third largest number of native speakers in India (84 million) thirteenth in the Ethnologies list of most-spoken languages worldwide, and most spoken Dravidian language. It is one of the twenty-two scheduled languages of the Republic of India and one of the four classical languages. The Telugu alphabet consist of 60 symbols - 16 vowels, 3 vowel modifiers, and 41 consonants. Sanskrit and Telugu alphabets are similar and exhibit one-one correspondence. Telugu has complete set of letters which follows scientific system to express sounds. Some of them are introduced to express fine shades of difference in sounds. Most of the positional base 10 numeral systems in the world have originated from India, where the concept of positional numerology was first developed. The Indian numeral system is commonly referred to in the West as the Hindu-Arabic numeral system or even Arabic numerals, since it reached Europe through the Arabs. Fig 1 below presents a listing of the symbols used in Telugu scripts for the numbers from zero to nine. 


\begin{tabular}{|l|l|l|l|l|l|l|l|l|l|}
\hline 0 & 1 & 2 & 3 & 4 & 5 & 6 & 7 & 8 & 9 \\
\hline$\bigcirc$ & $\bigcirc$ & 9 & 3 & $\mho$ & $\mho$ & $\varepsilon$ & $己$ & $\mho$ & $\Xi$ \\
\hline
\end{tabular}

Fig 1 Telugu numerals from 0 to 9

To the best of our knowledge standard dataset for printed Telugu numerals is not available till today. Therefore, dataset of printed Telugu numerals (isolated) 0 to 9 is created. The procedure followed is explained below. Printed numerals come in multi-font styles and sizes. To create the database of printed numerals, multi-font style and multi size numerals were chosen from Anu Script manager 4.0, Anu Script manager 5.0, Anu Script manager 6.0 and Anu Script manager 7.0, software packages. Numerals of 10 different font-styles namely Akshara Unicode, Pothana2000, Code2000, Gautami, RaghuTelugu, Saraswati5 and Vemana2000, Telugu numbers bold, telugu numbers thin, kasturi, vijaya,and Akshara-01 of 11 different font sizes 14, 16, 18, 20, 22, 24, 26, 28, 36, 48, 60, and 72 were collected. Apart from these, samples of machine printed numerals ( 25 pages) were scanned from printed Telugu text books. A total of 3150 images representing machine printed Telugu numerals were obtained and stored as data set.

\subsection{Preprocessing Method}

The recognition of handwritten numerals can achieve high performance based on pre processing stage also. The first step in preprocessing is to binarize the printed numeral image so that the numeral image has pixel values 0 and 1 . A thresholding application has to be performed on scanned gray scale images. We used Otsu's method [21] for the purpose of selecting the threshold and binarizing the gray scale images, so that resulting image has 0 as background pixels and 1 as foreground pixels. While converting the images into binary, images may have surplus elements one's (black) at undesirable places in the background image. This noise is to be removed from the binary digital image. To remove these unwanted one's from the background, noise removal algorithm is used. In this algorithm $3 \times 3$ templates are used. It is assumed that the pixel $(p, q)$ as center pixel and neighbors of the point $(p, q)$ are $(p-1, q)$, $(p-1, q+1),(p, q+1),(p+1, q+1),(p+1, q),(p+1, q-1),(p, q-1)$, and $(p-1, q-1)$, as is shown in Fig 2 .

\begin{tabular}{|l|l|l|}
\hline$p-1, q-1$ & $p-1, q$ & $p-1, q+1$ \\
\hline$p, q-1$ & $p, q$ & $p, q+1$ \\
\hline$p+1, q-1$ & $p+1, q$ & $p+1, q+1$ \\
\hline
\end{tabular}

Noise removal in an image depends on the type of noise in that image. To remove the surplus elements in Telugu printed numerals, the patterns which are shown in figures 3, 4, 5, and 6 are well suited for removing noise in binary digit images.

\begin{tabular}{|l|l|l|}
\hline 0 & 0 & 0 \\
\hline 0 & 1 & 0 \\
\hline 0 & 0 & 0 \\
\hline
\end{tabular}

Fig 2 Isolate pixel pattern.

\begin{tabular}{|l|l|l|}
\hline 0 & 0 & 0 \\
\hline 0 & 1 & 1 \\
\hline 0 & 0 & 0 \\
\hline
\end{tabular}
\begin{tabular}{|l|l|l|l|}
\hline 0 & 0 & 1 \\
\hline 0 & 1 & 0 \\
\hline 0 & 0 & 0 \\
\hline 0 & 1 & 0 \\
\hline 0 & 0 & 0 \\
\hline 0
\end{tabular} \mid \begin{tabular}{|l|l|l|l|l|}
\hline 0 & 0 & 0 \\
\hline 0 & 1 & 0 \\
\hline 1 & 1 & 0 \\
\hline 0 & 0 & 0 \\
\hline 0 & 0 \\
\hline 0 & 0 \\
\hline 0 & 0 & 0 \\
\hline 1 & 0 & 0 \\
\hline
\end{tabular}

\begin{tabular}{|l|l|l|}
\hline 0 & 0 & 0 \\
\hline 0 & 1 & 0 \\
\hline 0 & 1 & 0 \\
\hline
\end{tabular} \begin{tabular}{|l|l|l|}
\hline 0 & 0 & 0 \\
\hline 0 & 1 & 0 \\
\hline 0 & 0 & 1 \\
\hline
\end{tabular}

Fig 3 Two pixel patterns for noise removal 


\begin{tabular}{l}
\begin{tabular}{|l|l|l|}
\hline 0 & 0 & 0 \\
\hline 0 & 1 & 1 \\
\hline 0 & 0 & 1 \\
\hline
\end{tabular}$\quad$\begin{tabular}{|l|l|l|}
\hline 0 & 1 & 0 \\
\hline 0 & 1 & 1 \\
\hline 0 & 0 & 0 \\
\hline 1 & 0 & 0 \\
\hline 1 & 1 & 0 \\
\hline 0 & 0 & 0 \\
\hline
\end{tabular}$\quad$\begin{tabular}{|l|l|l|}
\hline 0 & 1 & 0 \\
\hline 1 & 1 & 0 \\
\hline 0 & 0 & 0 \\
\hline 1 & 1 & 0 \\
\hline 0 & 1 & 0 \\
\hline 0 & 0 & 0 \\
\hline
\end{tabular} \\
\hline \begin{tabular}{ll|l|}
$\mid 0$ & 0 & 0 \\
\hline 1 & 1 & 0 \\
\hline 1 & 0 & 0 \\
\hline
\end{tabular}
\end{tabular}

\begin{tabular}{|l|l|l|}
\hline 0 & 0 & 0 \\
\hline 1 & 1 & 0 \\
\hline 0 & 1 & 0 \\
\hline
\end{tabular}$\quad$\begin{tabular}{ll|l|l|}
\hline 0 & 0 & 0 \\
\hline 0 & 1 & 0 \\
\hline 0 & 1 & 1 \\
\hline
\end{tabular}

Fig 4 Three pixel patterns for noise removal

\begin{tabular}{|l|l|l|}
\hline 1 & 1 & 0 \\
\hline 1 & 1 & 0 \\
\hline 0 & 0 & 0 \\
\hline
\end{tabular}$\quad$\begin{tabular}{ll|l|l|}
\hline 0 & 1 & 1 \\
\hline 0 & 1 & 1 \\
\hline 0 & 0 & 0 \\
\hline
\end{tabular}

Fig 5 Four pixel patterns for noise removal

A total of 3150 numeral images representing Telugu machine printed 0 to 9, respectively, are obtained and stored as data set. Each numeral image represents a numeral (binary 1) that is unconstrained, isolated and clearly discriminated from the background (binary 0). The block diagram of preprocessing step is shown in the Fig 7.

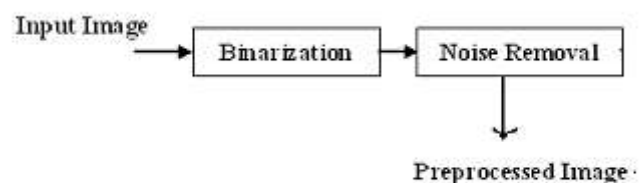

Fig 6 Preprocessing method

\section{FEATURE EXTRACTION}

Extraction of potential feature is an important component of any recognition system. To achieve high recognition performance depends on selection of potential features. The traditional goal of the feature extractor is to characterize an object by making numerical measurements. Good features are those whose values are similar for objects belonging to the same category and distinct for objects in different categories. In this paper, structural features, skeleton feature are considered as the potential features for the recognition of Telugu printed numerals. A brief overview of number of contours in a digit image, endpoint calculation, number of water reservoir areas, sides of the reservoir areas in the digits and also calculate the Ratio of Top line length to bottom line length are given below.

\subsection{Number of contours in an image}

The number of contours ( $\mathrm{NoC}$ ) is a structural feature. There are many techniques to find the number of contours in an image in systems of computer vision, some formats of coding of a contour are used - the Freeman chain code [11], twodimensional coding [ 12], polygonal coding, and the connected component labeling algorithm are most known. The contour is a boundary of object, a population of points (pixels), and separating object from a background. The contour contains the necessary information on the object shape. We use the connected component labeling algorithm[25] to find the number of contours. The number of background components (white components) minus one is the number of contours. For example, Telugu Printed digits $0,2,4$ have two contours and remaining digits have one contour only. The number of contours of the digits from 0to 9 shown in table1.

Table 1. Number of contours of each Digit

\begin{tabular}{|l|l|l|l|l|l|l|l|l|l|l|}
\hline Digit & 0 & 1 & 2 & 3 & 4 & 5 & 6 & 7 & 8 & 9 \\
\hline NoC & 2 & 1 & 2 & 1 & 2 & 1 & 1 & 1 & 1 & 1 \\
\hline
\end{tabular}

\subsection{Skeleton feature}

Thinning is usually a pre-processing stage in character recognition where the character image is reduced to a simplified one-pixel wide skeleton. In this paper use Fast thinning algorithm which gives good skeletons for digit images [22] for finding the Skeleton feature: Endpoint (EP). 


\section{Thinning:}

A binary digitized picture is defined by a matrix IT where each pixel IT(i, j) is either 1 or 0 . The pattern consists of those pixels that have value 1. Each stroke in the pattern is more than one element thick. Iterative transformations are applied to matrix IT point by point according to the values of a small set of neighboring points. It is assumed that the neighbors of the point $(i, j)$ are $(i-1, j),(i-1, j+1),(i, j+1),(i+1, j+1),(i+1, j),(i+1, j-1),(i, j-1)$, and $(i-1, j-1)$, as is shown in Figure 2.

\begin{tabular}{|c|c|c|}
\hline$\underset{(1-1 . j-1)}{P 0}$ & $\begin{array}{c}P 2 \\
(1-1, j)\end{array}$ & $\underset{(i-1, j+1)}{\text { P9 }}$ \\
\hline $\begin{array}{c}P B \\
0,-1)\end{array}$ & $\begin{array}{l}p 1 \\
\text { (a.j) }\end{array}$ & $\begin{array}{c}P 4 \\
\{, j+1\}\end{array}$ \\
\hline $\begin{array}{c}P 7 \\
(1-1, j-1)\end{array}$ & $\begin{array}{c}P 6 \\
(1+1 . j)\end{array}$ & $\begin{array}{c}\text { P5 } \\
(1+1, j+1)\end{array}$ \\
\hline
\end{tabular}

Fig 2 Designations of the nine pixels in a $3 \times 3$ window

The new value given to a point at the $\mathrm{n}^{\text {th }}$ iteration depends on its own value as well as those of its eight neighbors at the $(n-1)^{\text {th }}$ iteration, so that all digit points can be processed simultaneously. It is assumed that a $3 \times 3$ window is used, and that each element is connected with its eight neighboring elements. The algorithm [18] is requires only simple computations.

\section{Parallel Thinning Algorithm: It consists of two Sub iterations}

Step1: deleting the south-east boundary points and north-west corner points

Step2: north-west boundary points and south-east corner points are deleted

Step3: end points and pixel connectivity are preserved

The above procedure is done until there are no changes in the image

\section{Procedure:}

In order to preserve the connectivity of the skeleton, divide each iteration into two sub iterations. In first iteration, the contour pixel P1 is deleted from pattern if the following conditions are satisfied

(i) $2<=\mathrm{B}(\mathrm{P} 1)<=6$

(ii) $\mathrm{A}(\mathrm{P} 1)=1$

(iii) $\mathrm{P} 2$ * $\mathrm{P} 4$ * $\mathrm{P} 6=0$

(iv) $\mathrm{P} 4{ }^{*} \mathrm{P} 6{ }^{*} \mathrm{P} 8=0$

In second Iteration, the contour pixel P1 is deleted from pattern if the following conditions are satisfied

(i) $2<=\mathrm{B}(\mathrm{P} 1)<=6$

(ii) $A(P 1)=1$

(iii) $\mathrm{P} 2{ }^{*} \mathrm{P} 4$ * $\mathrm{P} 8=0$

(iv) $\mathrm{P} 2$ * $\mathrm{P} 6$ * $\mathrm{P} 8=0$

Where $A(P 1)$ is the - The number of 01 patterns in ordered set $P 2, P 3, P 4 \ldots P 8, P 9$ that are 8 neighbors of $P 1$ (figure 9 ) and $\mathrm{B}(\mathrm{Pi})$ is the number of nonzero neighbors of $\mathrm{P} 1$, that is, $\mathrm{B}(\mathrm{P} 1)=\mathrm{P} 2+\mathrm{P} 3+\mathrm{P} 4+\cdots+\mathrm{P} 8+\mathrm{P} 9$. If any condition is not satisfied, e.g., the values of P2, P3, P4, - - P9 as shown in Figure 10, then $A(P i)=2$ Therefore, $P 1$ s not dele ted from the digit picture. The Result of the above algorithms in shown in figure 11 ,

\begin{tabular}{|l|l|l|}
\multicolumn{4}{|c|}{$/ 1$} \\
\hline 0 & 0 & 1 \\
\hline 1 & $p$ & 0 \\
\hline 1 & 0 & 0 \\
\hline \multicolumn{2}{|c}{}
\end{tabular}

Fig 3 Counting the 01 patterns in the ordered set P2, P3, P4, ... P8, P9. 


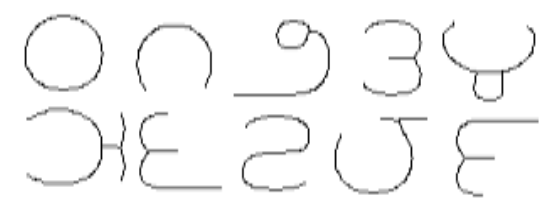

Fig 4 Thinned images of Fig1

By using the skeleton(single pixel width) of the digit's body to extract one feature: feature point.

Feature point: One important feature point can be easily found from the skeleton by examining the eight immediate neighbors of every black pixel $p$ shown in figure 6 .

End point: End point (EP) [20] is a digit point (black pixel) with only one black neighbor. The patterns used to find endpoints of an image when the image is scan from top left corner to bottom right corner in shown in figure 12. The table2 shows the number of end points of the each Telugu numerical digit. The figure 12 shows the end points of each telugu numeral digit

\begin{tabular}{|l|l|l|}
\hline 0 & 0 & 0 \\
\hline 0 & 1 & 1 \\
\hline 0 & 0 & 0 \\
\hline
\end{tabular} \mid \begin{tabular}{|l|l|l|}
\hline 0 & 0 & 1 \\
\hline 0 & 1 & 0 \\
\hline 0 & 0 & 0 \\
\hline
\end{tabular} \begin{tabular}{|l|l|l|}
\hline 0 & 1 & 0 \\
\hline 0 & 1 & 0 \\
\hline 0 & 0 & 0 \\
\hline
\end{tabular}

\begin{tabular}{|l|l|l|}
\hline 1 & 0 & 0 \\
\hline 0 & 1 & 0 \\
\hline 0 & 0 & 0 \\
\hline
\end{tabular}$\left|\begin{array}{|l|l|l|}\hline 0 & 0 & 0 \\
\hline 1 & 1 & 0 \\
\hline 0 & 0 & 0 \\
\hline\end{array}\right|$\begin{tabular}{ll|l|l|}
\hline 0 & 0 & 0 \\
\hline 0 & 1 & 0 \\
\hline 1 & 0 & 0 \\
\hline
\end{tabular}

\begin{tabular}{|l|l|l|}
\hline 0 & 0 & 0 \\
\hline 0 & 1 & 0 \\
\hline 0 & 1 & 0 \\
\hline
\end{tabular} \begin{tabular}{|l|l|l|}
\hline 0 & 0 & 0 \\
\hline 0 & 1 & 0 \\
\hline 0 & 0 & 1 \\
\hline
\end{tabular}

Fig 5 end point patterns.

Table 2. Endpoints count of the Each Digit

\begin{tabular}{|l|l|l|l|l|l|l|l|l|l|l|}
\hline Digit & 0 & 1 & 2 & 3 & 4 & 5 & 6 & 7 & 8 & 9 \\
\hline EP & 0 & 2 & 1 & 3 & 2 & 4 & 3 & 2 & 3 & 3 \\
\hline
\end{tabular}

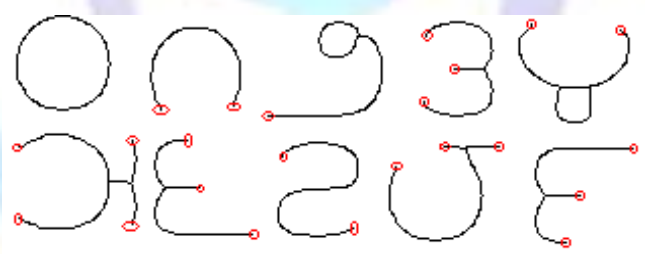

Fig 6 End points of Digit Images

3.3Number of Water Reservoir Sides and Water Reservoir side of the Image:

Number of Water Reservoirs side

The water reservoir principle is as follows. If water is poured from a side of a component, the cavity regions of the component where water will be stored are considered as reservoirs [23]. The cavity regions of the components where water will be stored are considered as reservoir. Telugu printed digits generate reservoirs which are used for recognition. Now, we will discuss the water reservoir extraction scheme of Telugu printed Digits.

Top reservoir: By top reservoirs of a digit, we mean the reservoirs obtained when water is poured from top of the digit. Bottom reservoir: By bottom reservoirs of a digit, we mean the reservoirs obtained when water is poured from bottom of the digit. A bottom reservoir of a digit is visualized as a top reservoir when water will be poured from top after rotating the digit image by 180 degrees. Left (right) reservoir: If water is poured from the left (right) side of a component, the cavity regions of the digit where water will be stored are considered as left (right) reservoirs. The figure 14 illustrates the reservoirs. Number of sides water will preserve in a digit is considering as one of the feature for recognition. For Recognizing the Telugu digits 1 and 7 , use this feature. Digit 1 has one side and 7 have two sides. The Fig shows the Reservoir areas. 


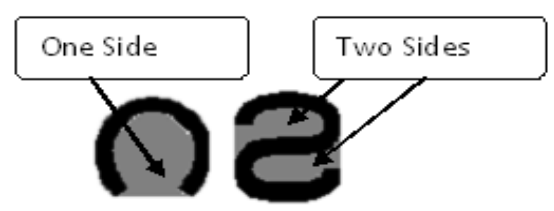

Fig 7 Reservoir areas of digit 1 and 7

For recognizing the digits 3, 6, 8 and 9, use the sides of water reservoir area. The digit 3 has LEFT side, the Digits 6 and 9 have RIGHT side and the Digit 8 has TOP side.

\subsection{Ratio of Top Line to Bottom Line length of the Digit}

The ratio length of the top line $(\mathrm{tl})$ to length of the bottom line (bl) in the digit image is consider as a feature for recognize the Digits 6 and 9 . The figure 15 sow $s$ the lengths of the top line and bottom line of the digits 6 and 9 .

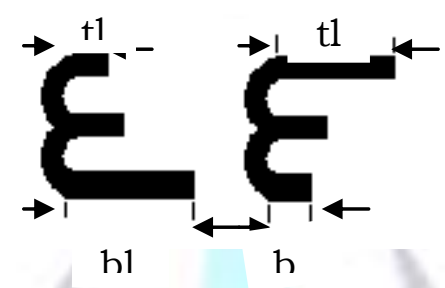

Fig 8 Calculation of top line and bottom line.

\section{RECOGNITION PROCESS}

For recognition a digit, first find out the number of contours. Based on these feature the digit is classified as either Group1 or Group2. For classifying the Group1 digits, calculate the number of Endpoints (EP) of the image. If NEP is zero then the Digit is classified as Digit 0 , If EP is one then the Digit is classified as Digit 2, If EP is two then the Digit is classified as Digit 4 in Grop1. For classifying the Group2 digits, calculate the number of Endpoints (EP) of the image. Based on these feature Group2 digits are classified as Group2a, Group2b and digit 5. Grop2a consists of the digits 1 and 7. Grop2b consists of the digits 3,6,8 and 9. For classifying the digits in Group2a calculate the number of sides of the reservoir is occurred in the image. For Digit 1 the number of sides of the reservoir is 1 , and for Digit 7 the number of sides of the reservoir is 2 . For classifying the digits in Group2b calculate side of the reservoirs in the image. Based on these feature two digits are classified. The side of the reservoir for digit 3 is LEFT, for the digit 8 is TOP, and the digits 6 and 9 are RIGHT. For classifying the Digits 6 and 9 find the ratio of the length of top line(tl) to length of the bottom line (bl). The ratio is grater than 1 then digit is classified as 9 and otherwise classified as digit 6 . The flow chart for the recognition procedure is shown in figure16a and $b$.

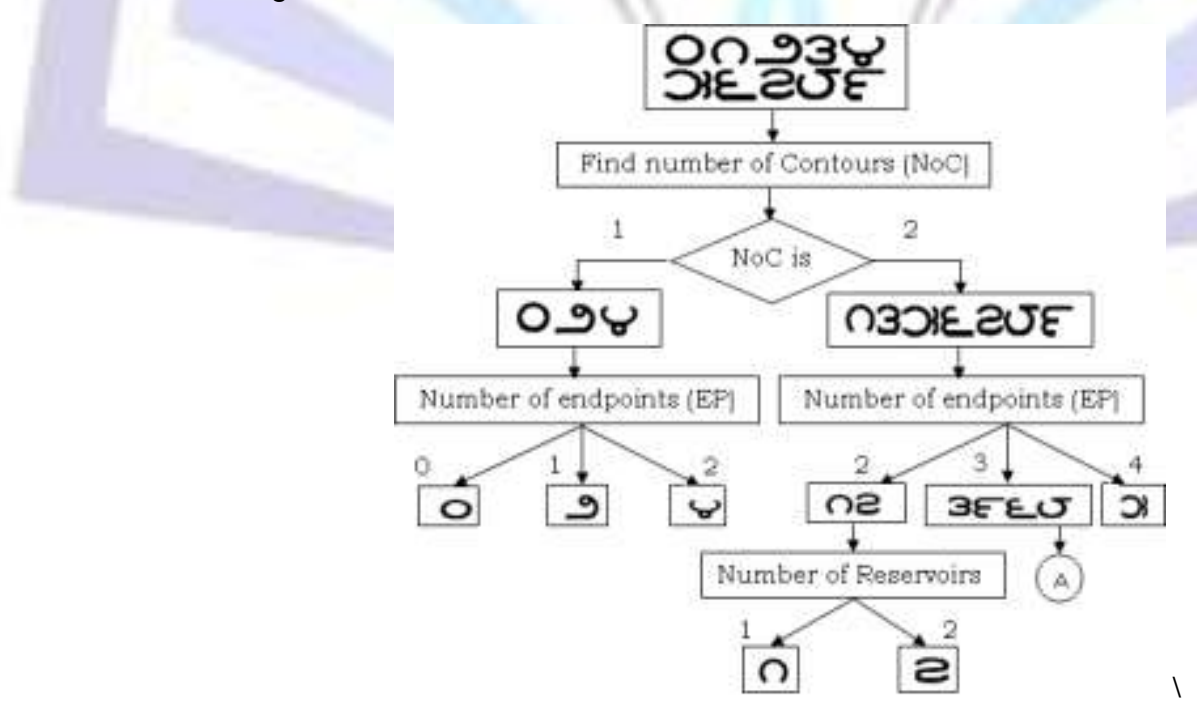

Fig 16 a 


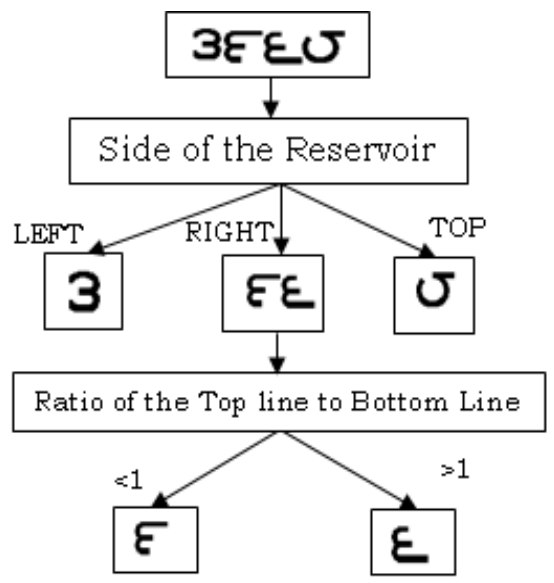

Fig $16 \mathrm{~b}$

Fig 15. (a) and (b) flowchart for the Recognition algorithm

The Algorithm for the recognition of Telugu printed numerals are shown below

Algorithm: Recognition of Telugu Digits

Input: Isolated numeral image

Output: Recognition of the Numeral

Method: Structural, Statistical features extraction.

Step1: Pre Process the image

Step2: Find the Number of Contours (NoC) and classify the digit as Group1 or Group2

Step3: Find out the Skeleton of input image and calculate the Skeleton feature number of end points(EP) in both Groups

Step4: Based on EP in Group1 Recognize the Digits 1, 2 \& 4

Step5: classify the Digits in Group1 based on EP.

Step6: classify the Digits in group2 based on EP into Group2a and Group2b and recognize the Digit 5.

Step7: estimate the number of sides of the reservoir in Group2a

Step8: Based on that feature classify the Digits 1 and 7

Step9: Find the Sides of the water reservoir in Group2b.

Step10: Based on these feature classify the Digits in Group2b into Group2b1 and recognize the digits 3 and 8

Step11: Calculate the ratio of length of top line to bottom line in the image

Step12: based on the above features Recognize the digit 6 and 9

\section{Experimental results}

In order to classify the telugu printed numeral, and evaluate the performance/consistency of the proposed method, Experiments were conducted to test the efficiency of our novel feature extraction method on 3150 different size telugu numerals. The proposed method was experimented on both machine printed numerals images obtained as described in section 2 . The proposed method recognizes the digits based on the feature at maximum 5 . Every digit does not need all these 5 features. The number of feature required to recognize the digits is shown in the table 3 . The $100 \%$ recognition rate is achieved in this method.

Table 3. number of features required for recognizes the Telugu Digit

\begin{tabular}{|c|c|c|c|c|c|c|c|c|c|c|}
\hline Digit & 0 & 1 & 2 & 3 & 4 & 5 & 6 & 7 & 8 & 9 \\
\hline NoFR & 2 & 3 & 2 & 4 & 2 & 2 & 5 & 3 & 4 & 5 \\
\hline
\end{tabular}

\section{Conclusions}

We have described an efficient method for recognition of machine printed Telugu numerals using only five simple features: Number of end points, Number of contours, Number of sides and sides of the water reservoir of the image and 
ratio of the length of top line to bottom line. Promising recognition results are obtained by combining these features. The experiments have been carried out on 3150 printed Telugu numeral images The recognition of $100 \%$ is achieved for Printed Numerals. The novelty of the proposed method is that no standard classification algorithms are used. The results obtained encourage us to continue the research to classify the Telugu handwritten numerals without any standard classification algorithm

\section{ACKNOWLEDGMENTS}

The authors would like to express their gratitude to Sri K.V.V. Satya Narayana Raju, Chairman, and K. Sashi Kiran Varma, Managing Director, Chaitanya group of Institutions for providing necessary infrastructure. Authors would like to thank anonymous reviewers for their valuable comments

\section{REFERENCES}

[1] A.F.R. Rahman, R.Rahman, M.C.Fairhurst, "Recognition of handwritten Bengali Characters: A Novel Multistage Approach", Pattern Recognition, 35,997-1006, 2002.

[2] A.L.Koerich, R. Sabourin, C.Y.Suen, "Large off-lineHandwritten Recognition: A survey", Pattern Analysis Application, 97-121, 2003.

[ 3 ] B. B. Chaudhuri and U. Pal, A complete printed Bangla OCR system, Pattern Recognition, vol. 31, pp. 531-549, (1998).

[ 4 ] B.V. Dhandra, R.G.Benne and Mallikargun Hangargi, "Isolated Handwritten Kannada Numeral recognition based on Template matching", IEEE-ACVIT -07, pp.1276-1282, Dec-2007.

[ 5] Cheng-Lin, Kazuki, Hiroshi, and Hiromichi, Handwritten Digit Recognition: Investigation of Normalization and Feature Extraction Techniques", Pattern Recognition, 37(2004), pp. 265-279.

[ 6 ] C.L. Liu, K. Nakashima, H. Sako, H. Fujisawa, Handwritten digit recognition: benchmarking of state-of the-art technique. Pattern Recogn. vol 36, pp 2271-2285(2003)

[ 7 ] Dhandra. B.V, Benne. R.G, Hangarge M, Handwritten Kannada Numeral Recognition Based on Structural Features. Proceedings of International Conference on Computational Intelligence and Multimedia Applications, vol 2, pp-224-228, (2007)

[ 8 ] Dinesh Acharya U, N V Subbareddy and Krishnamoorthy, Isolated Kannada Numeral Recognition Using Structural Features and K-Means Cluster, Proc. of IISN-2007, (2007) 125-129.

[ 9 ] E. Kussul and T. Baidyk, "Improved Method of Handwritten Digit Recognition Tested on MNIST Database", Image and Vision Computing, 22(2004), pp. 971-981.

[10] G.G.Rajput and Mallikarjun Hangarge , "Recognition of Isolated Kannada Numeral Based on Image Fusion Method", PReMI 2007, LNCS 4815, pp. 153-160, 2007.

[11] G. G. Rajput, Rajeswari Horakeri, Sidramappa Chandrakant "Printed and Handwritten Kannada Numeral Recognition Using Crack Codes and Fourier Descriptors Plate" RTIPPR, 2010. IJCA Special Issue on "Recent Trends in Image Processing and Pattern Recognition" pp.53-58 2010

[12] Graham, D.N "Image transmission by two-dimensional contour coding" Proceedings of the IEEE Volume: 55, Issue: 3 Pages: March 1967336 - 346

[13] Hanmandlu M, M.Hafizuddin, M Yusuf and V K Madasu , Fuzzy based Approach to recognition of multifont Numerals, Proc. Of $2^{\text {nd }}$ National Conf. on Document Analysis and Recognition (NCDAR), Mandya, vol., pp 118-126, (2003)

[14] Ivind due trier, anil Jain, torfiinn Taxt, "A feature extraction method for character recognition-A survey ", pattern Recg, vol 29, No 4, pp-641-662, 1996

[15] J.D. Tubes, A note on binary template matching. Pattern Recognition, 22(4):359-365, 1989.

[16] Nor Amizam Jusoh and Jasni Mohamad Zain "Application of Freeman Chain Codes: An Alternative Recognition Technique for Malaysian Car Plates" IJCSNS International Journal of Computer Science and Network Security, VOL.9 No.11, November 2009 222-227 pages

[17] P.Nagabhushan, S.A.Angadi, B.S.Anami, "A fuzzy statistical approach of Kannada Vowel Recognition based on Invariant Moments”, NCDAR-2003, Mandy, India, pp275-285, 2003.

[18] R. Chandrashekaran, M.Chandrasekaran, Gift Siromaney "Computer Recognition of Tamil, Malayalam and Devanagari characters", Journal of IETE, Vol.30, No.6, 1984.

[19] Reena Bajaj, Lipika Dey and Santanu Chaudhur, "Devnagari numeral recognition by combining decision of multiple connectionist classifiers", Vol. 27, Part 1, pp. 59-72, February 2002.

[20] Seong- W han Lee, "Multi layer cluster neural network for totally unconstrained handwriritten numeral recognition. Neural Networks. Vo I. 8. No 5. pp.409-4 1 8, 1984 
[21] S.V. Rajashekararadhya et.al "Efficient Zone Feature Extraction Algorithm for Handwritten Numerals Recognition of Four South Indian Scripts", Journal of Theoretical and Applied Information Technology 2008

[22] T. Y. ZHANG and C. Y. SUEN “A Fast Parallel Algorithm for Thinning Digital Patterns" Image Processing and Computer Vision, March 1984 Volume 27 Number 3

[ 23 ] U Pal and P.P.Roy, "Multi-oriented and curved text lines extraction from Indian documents", IEEE Trans on system, Man and Cybernetics-Part B, vol.34, pp.1667-1684, 2004.

[24] U. Pal, T. Wakabayashi, N. Sharma and F. Kimura,"Handwritten Numeral Recognition of Six Popular Indian Scripts", In Proc. 9th International Conference on Document Analysis and Recognition. pp. 749-753, Curitiba, Brazil, September 24-26, 2007.

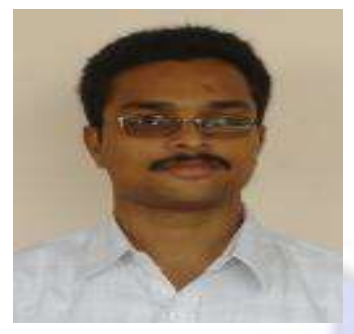

\section{Author' biography with Photo}

U Ravi Babu obtained his MSc Information Systems (IS) from AKRG PG College, Andhra University in the year 2003 and M.Tech Degree from RVD University in the year 2005. He is a member of SRRF-GIET, Rajahmundry. He is pursuing his Ph.D from AN University-Guntur in Computer Science \& Engineering under the guidance of Dr V. Vijaya Kumar. He has published research papers in various National, Inter National conferences, proceedings. He is working as an Assistant Professor in GIET, Rajahmundry from July 2003 to till date. He is a life member of ISCA
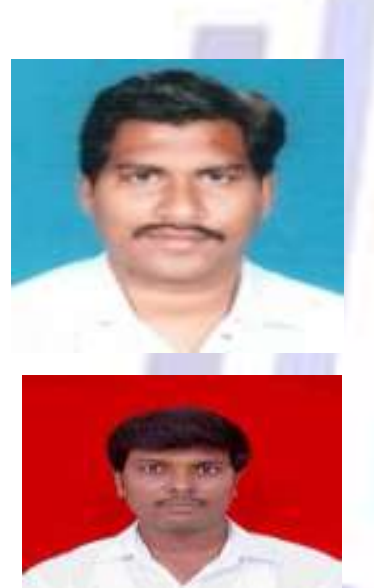

S.MaruthuPerumal received his Ph.D at Dr MGR University Chennai under the Guidance of Dr $\mathrm{V}$ VijayaKumar.He is having fifteen years of teaching experience. At present he is working as Professor and Head Department of CSE Godavari Institute of Engineering and Technology, Rajahmundry. His research interest includes Image processing, Digital Watermarking, Steganography and Security. He is a life member of ISCA,IAENG. 